\title{
La chloroquine : mécanisme d'action antimalarique et résistance
}

Quoique la chloroquine soit l'antimalarique le plus utilisé depuis de nombreuses décennies, son mode d'action sur les formes intra-érythrocytaires de Plasmodium reste mal connu. On sait cependant que l'effet schizonticide est lié à la concentration du médicament dans les hématies parasitées, et principalement les vacuoles digestives du parasite. Les propriétés basiques de la chloroquine ne suffisent pas à expliquer une telle concentration dans les compartiments acides des Plasmodium, et des phénomènes actifs de concentration via un transporteur membranaire semblent vraisemblables. La résistance à la chloroquine, qui s'étend dans le monde, est associée à la perte du pouvoir de concentration dans les vacuoles digestives; un transporteur chargé de l'élimination de cette molécule pourrait être responsable de ce phénomène, rappelant la résistance des cellules à certains cytolytiques via l'hyperactivité d'une glycoprotéine membranaire, produit du gène MDR (multidrug resistance).

\section{Serge Moreau}

\section{ADRESSE}

S. Moreau : chargé de recherche à l'Inserm. Inserm U. 42, 369, rue Jules-Guesde, 59650 Villeneuve-d'Ascq, France. a chimiothérapie est la seule arme efficace dont nous disposons actuellement dans la lutte contre cette endémie majeure que constitue le paludisme. La chloroquine (figure 1, p. 730 ) est probablement la molécule la plus utilisée tant en chimiothérapie qu'en prophylaxie. Son succès est lié à la rapidité de ses effets biologiques, à la très bonne tolérance que l'on peut observer aux doses utilisées en prophylaxie et à un bas prix de revient. L'apparition de la résistance en Asie du Sud-Est et en Amérique du Sud, son extension au cours de la dernière décennie au continent africain, constituent une menace sérieuse pour son utilisation (figure 2, p. 731) [1]. La synthèse et le criblage de plus de 200000 molécules n'ont permis de faire émerger que quelques antimalariques potentiels. La méfloquine (figure 1, p. 730), issue de cette étude, est actuellement une des meilleures alternatives à la disposition du thérapeute. Ses parentés structurales avec la quinine, la relative facilité avec laquelle il est possible de développer expérimentalement une résistance à cette molécule, en particulier chez les souches déjà résistantes à la chloroquine, montrent qu'il est urgent de concevoir et de rechercher de nouvelles séries structurales. La compréhension des mécanismes d'action de la chloroquine, ou des mécanismes développés par le parasite pour résister aux médicaments, peut constituer une aide efficace pour la conception des médicaments de l'avenir.

Peu de travaux ont été consacrés à une telle approche, et nos connaissances dans ce domaine restent limitées. Cette brève revue tentera de faire 
1. Payne D. Spread of chloroquine resistance in Plasmodium falciparum. Parasitology Today $1987 ; 3: 241-6$.

2. Slomianny C, Prensier G, Charet P. Ingestion of erythrocytic stroma by Plasmodium chabaudi trophozoites: ultrastructural study by serial sectioning and 3-dimensional reconstruction. Parasitology 1985 ; 90 : 579-88.

3. Slomianny $\mathrm{C}$. Thèse ès sciences, université de Lille, 1984.

4. Fitch CD, Yunis NG, Chevli R, Gonzalez Y. High-affinity accumulation of chloroquine by mouse erythrocytes infected with Plasmodium berghei. J Clin Invest 1974 ; 54 : 24-33.

5. Cohen SN, Yielding KL. Spectrophotometric studies of the interaction of chloroquine with deoxyribonucleic acid. J Biol Chem 1965 ; 240 : 3123-31.

6. Chou AC, Chevli R, Fitch CD. Ferriprotoporphyrin IX fulfills the criteria for identification as the chloroquine receptor of malaria parasites. Biochemistry 1980 ; 19 : 1543-9.

7. Moreau S, Perly B, Biguet J. Interaction of chloroquine with ferriprotophorphyrin IX. Nuclear magnetic resonance study. Biochimie $1982 ; 64: 1015-25$.

8. Mackenzie AH, Scherbel AH. Chloroquine and hydroxychloroquine in rheumatological therapy. Clin Rheum Dis 1980; 6: 545-66.

9. De Duve C, De Barsy T, Poole B, Trouet A, Tulkens $P$, Van Hoof $F$. Lysosomotropics agents. Biochem Pharmacol 1974; 23 : 2495531.

10. Homewood CA, Warhurst DC, Peters W, Baggaley VC. Lysosomes, $\mathrm{pH}$ and the antimalarial action of chloroquine. Nature 1972 ; $235: 50-2$.

11. Krogstad DJ, Schlesinger PH, Gluzman IY. Antimalarials increase vesicle $\mathrm{pH}$ in Plasmodium falciparum. J Cell Biol 1985 ; $101: 2302-9$

12. Mahmalgi J, Veignie E, Prensier G, Moreau S. Relations between resistance to chloroquine and acidification of endocytic vesicles of Plasmodium berghei. Parasitology $1989 ; 98: 1-6$.

13. Yayon A, Cabantchik ZI, Ginsburg H. Identification of the acidic compartment of Plasmodium falciparum-infected human erythrocytes as the target of the antimalarial drug chloroquine. EMBO J $1984 ; 3$ : 2695 700 .

14. Moreau S, Prensier G, Maalla J, Fortier B. Identification of distinct accumulation sites of 4-aminoquinoline in chloroquine sensitive and resistant Plasmodium berghei

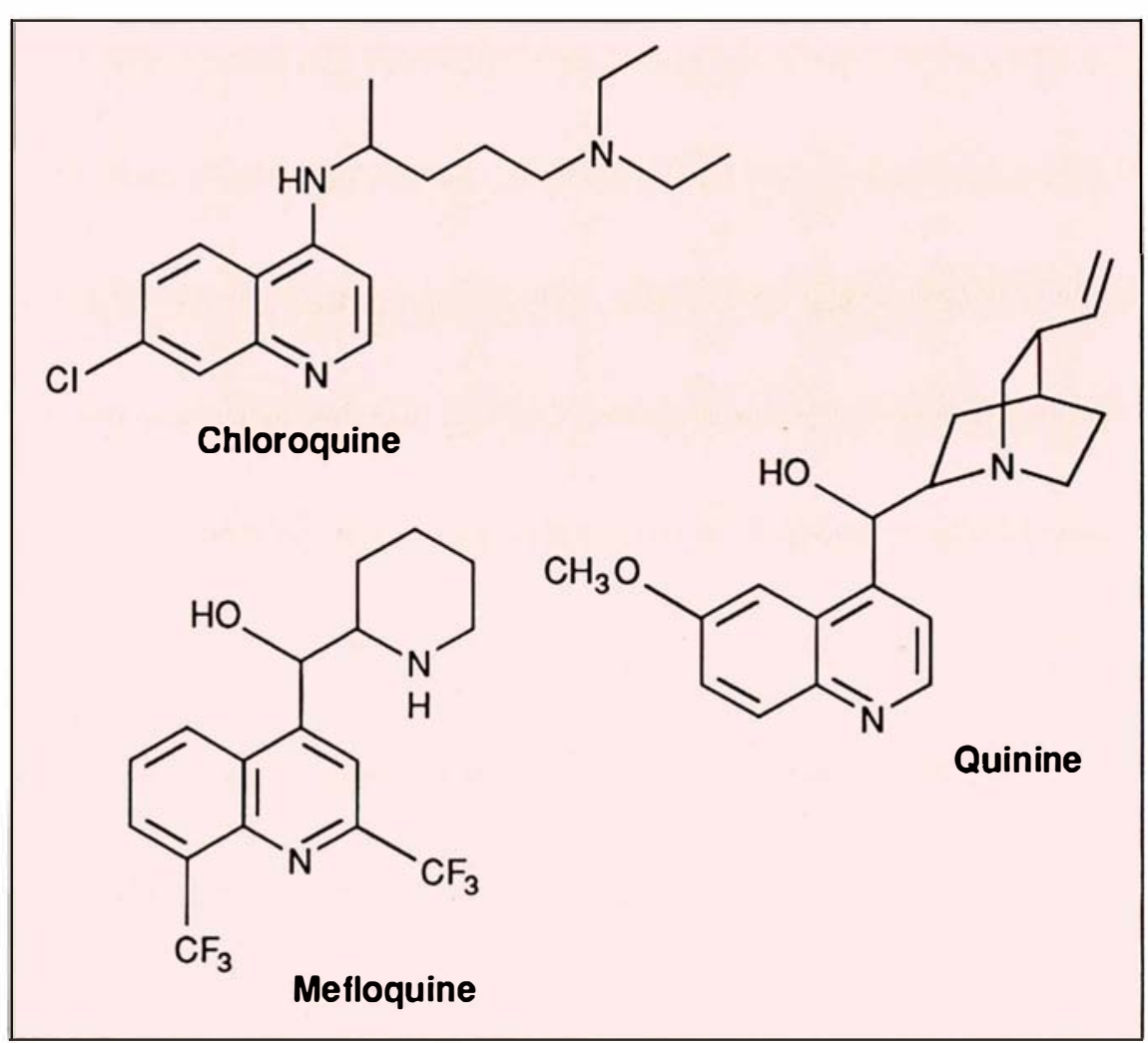

Figure 1. Structures chimiques des principaux schizonticides sanguins.

le point, en soulignant les progrès récents laissant entrevoir de nouvelles perspectives.

\section{Effets biologiques}

La chloroquine (comme la quinine) agit uniquement au niveau du stade intra-érythrocytaire du parasite. L'importance de la dégradation de l'hémoglobine dans la sensibilité du Plasmodium à cette série de molécules est bien connue. Les données biologiques soulignent en effet les relations existant entre l'activité des schizonticides sanguins (chloroquine, quinine, etc.), les stades parasitaires et les activités protéolytiques du protozoaire vis-à-vis de cette hémoprotéine. Chez certaines espèces, le gamétocyte est également sensible à ces drogues, en particulier quand ce stade est immature et dégrade encore l'hémoglobine. Les gamétocytes matures n'y sont jamais sensibles. La connaissance des structures parasitaires liées à cette dégradation est donc nécessaire pour étudier l'impact de la chloroquine (et des schizonticides sanguins) sur la croissance de Plasmodium dans l'érythrocyte.
Des travaux de microscopie électronique associés à une analyse cytochimique de quelques enzymes intervenant dans la dégradation de l'hémoglobine ont permis de mettre en évidence un système complexe d'endocytose du cytoplasme érythrocytaire par le parasite [2]. Il associe un système cytostomal ${ }^{*}$ et un système de micropinocytose ${ }^{* *}$ dont l'importance relative est fonction de

* Système cytosomal : système appartenant au processus général d'endocytose. Il est cependant particulier aux sporozoaires car il implique l'existence d'une structure spéciale qui est une différenciation de la membrane plasmique: le cytostome (ou micropore). La membrane plasmique s'invagine à partir de cette structure en entraînant de grandes portions de cytoplasme de l'hématie de façon à former des vacuoles puis des tubes cytostomaux dans lesquels va se produire la dégradation de l'hémoglobine.

** Micropinocytose: processus qui, avec la phagocytose, fait partie du phénomène d'endocytose. Ce dernier permet à la cellule d'ingérer des macromolécules, des particules ou des microorganismes. La pinocytose est le phénomène par lequel la cellule internalise des liquides ou des solutés. Dans le cas du parasite, la micropinocytose conduit à l'ingestion de petites portions de cytoplasme érythrocytaire dans des vésicules dont le contenu sera dégradé. 


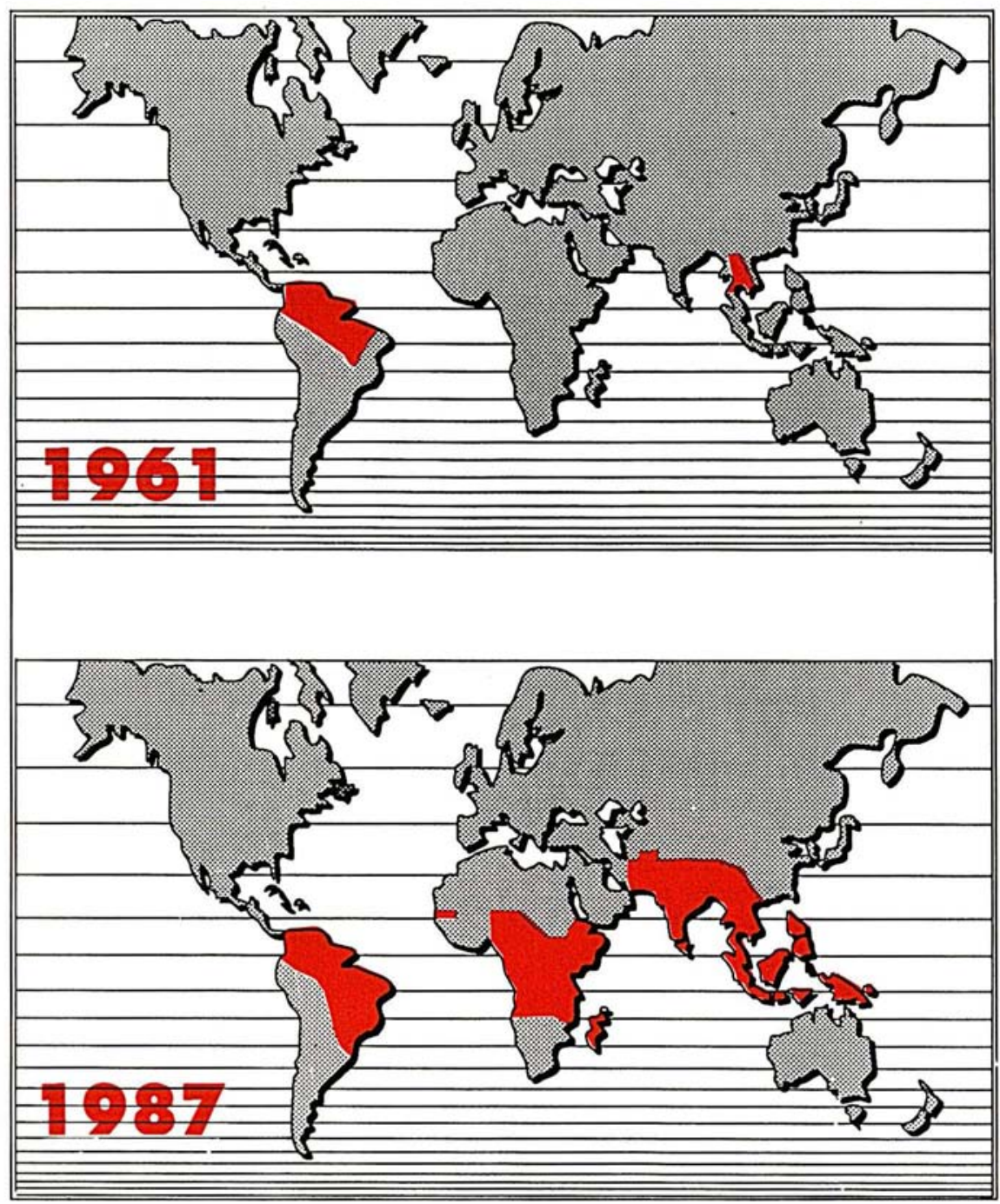

Figure 2. Extension des zones à Plasmodium falciparum chloroquinorésistant entre 1961 et 1987. Les deux foyers initiaux (Colombie et frontière Cambodge-Thailande) se seraient manifestés dès 1957. Un foyer africain, apparemment indépendant des précédents, est apparu sur la côte est vers 1977. (D’après [1].)

la maturité de l'organisme.

La dégradation de l'hémoglobine dans ces structures spécialisées conduit à la formation du pigment malarique : association de protéines et de porphyrines dont la nature précise est mal connue. La présence de grains de pigment dans les vacuoles d'endocytose parasitaires est une bonne indication de l'activité protéolytique de ces structures. Cette pigmentation permet de suivre par microscopie optique la maturation du parasite, elle est une aide pré$\mathrm{m} / \mathrm{s} n^{\circ} 10$ vol. 5 , décembre 89 des parasites intra-érythrocytaires. Une analyse ultrastructurale montre que cette fusion se traduit par l'incorporation des vacuoles de pinocytose dans une grande vacuole autophage, où les processus de digestion de l'hémoglobine ont été bloqués (figure 3, p. 732) [3]. Cet effet est spécifique des amino-4 quinoléines et n'est pas observé pour la quinine.

\section{Concentration intraparasitaire}

Les propriétés antimalariques des schizonticides sanguins sont liées à la concentration préférentielle de ces molécules dans le parasite intra-érythrocytaire. Dans les hématies parasitées, la chloroquine peut atteindre des concentrations qui sont plusieurs centaines de fois plus élevées que celles présentes dans les hématies normales [4]. On peut mesurer, pour ce phénomène, une constante d'affinité de $10^{8} \mathrm{M}^{-1}$. Il est saturable et dépend des fonctions énergétiques de la cellule, il peut être inhibé par des molécules de la même classe thérapeutique. La recherche d'un récepteur cellulaire responsable de la concentration intraparasitaire des amino-4 quinoléines a constitué un objectif constant pour les équipes engagées dans cette voie. Cet objectif paraît d'autant plus important que l'analyse de la résistance conduit à montrer une chute de l'affinité de la chloroquine pour la plupart des souches résistantes étudiées.

L'activité antimalarique de la chloroquine est, par ailleurs, liée à des paramètres structuraux bien définis. Les relations entre structure chimique et activité biologique ont été parfaitement étudiées grâce à la synthèse de nombreux dérivés. Ces études mettent en évidence le rôle essentiel des propriétés de base faible de cette série de molécules. La chloroquine possède deux azotes basiques de $\mathrm{pKa}^{* * *} 10,2$ et 8,1 (figure 1) dont la présence est indispensable à l'expression de son activité biologique. L'atome de chlore en position 7 pigmentaires en une ou deux grosses vacuoles résiduelles aisément mises en évidence en microscopie. Ce clumping apparaît très rapidement après administration de la drogue; en 80 minutes, chez un Plasmodium de rongeur (P. berghei), une dose unique de chloroquine suffit à provoquer la fusion des vacuoles digestives
*** pKa: échelle de mesure de la force d'un acide ou d'une base dans l'eau définie à partir de l'équilibre: $\mathrm{AH}+\mathrm{H}_{2} \mathrm{O} \rightleftharpoons \mathrm{H}_{3} \mathrm{O}^{+}+A^{-}$; $\mathrm{Ka}=\left[\mathrm{H}_{3} \mathrm{O}^{+}\right]\left[\mathrm{A}^{-}\right] /[\mathrm{AH}], \mathrm{pKa}=-\log \mathrm{Ka}$. Plus le pKa est grand, plus la base $A$-est forte (échelle variant de 0 à 14). 

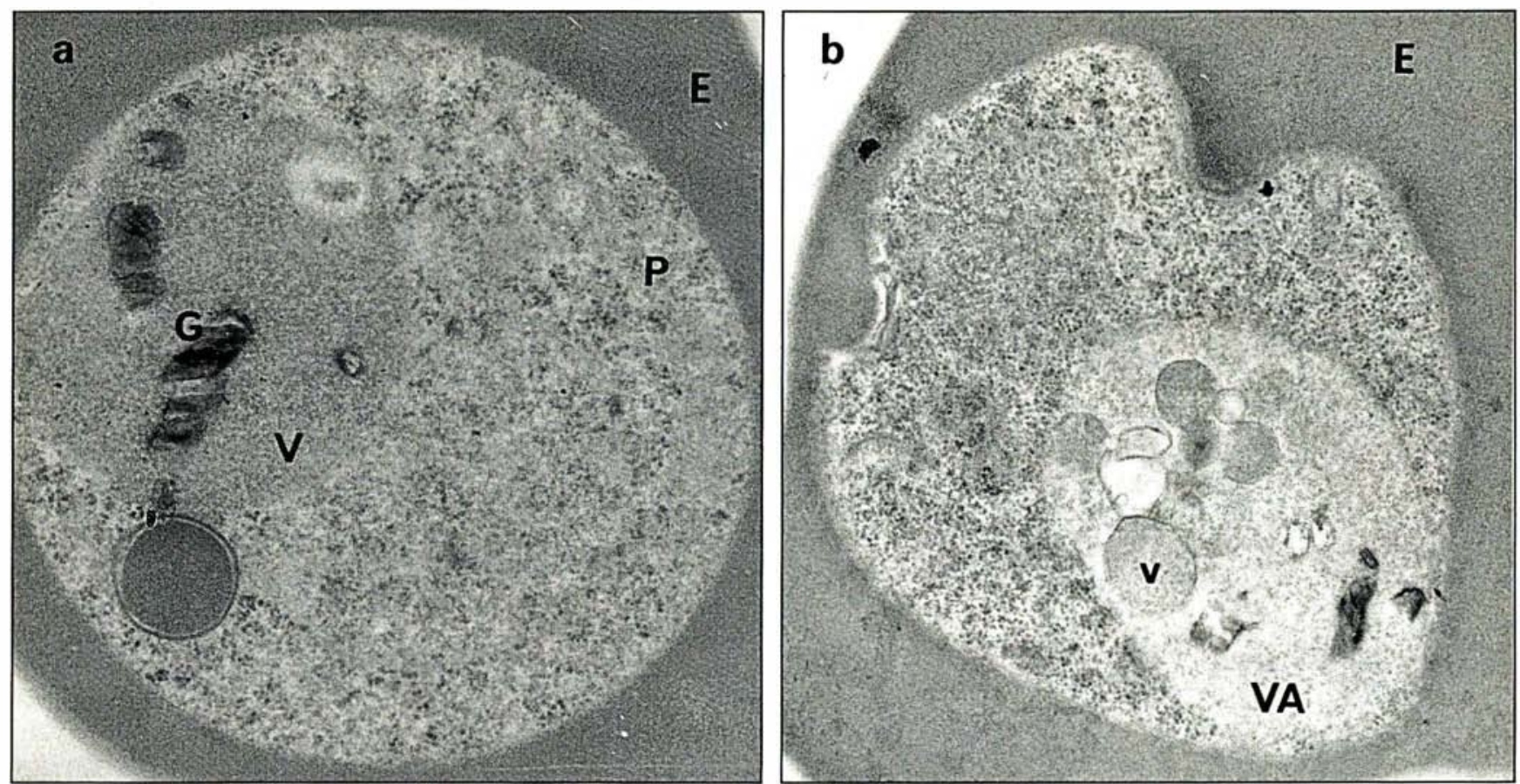

Figure 3. Effet biologique de la chloroquine sur Plasmodium falciparum en culture. a. Érythrocyte parasité par $P$. falciparum $(\times 30000)$. $E=$ érythrocyte; $P=$ parasite $;=$ vacuole digestive $; G=$ grain de pigment, résidu de dégradation de l'hémoglobine. b. Érythrocyte parasité (X21 000) après traitement par la chloroquine $(2.10-8 \mathrm{M}, 60 \mathrm{~min})$. $E=$ érythrocyte $V A=$ vacuole autophage $; v=$ vésicule d'endocytose non dégradée traduisant l'inhibition des activités enzymatiques de la vacuole par la chloroquine. (Documents communiqués par C. Slomianny, Inserm U. 42.)

de la quinoléine (figure 1) est également un élément clé de cette activité ; sa simple substitution par un atome d'hydrogène entraîne une chute de plus de $90 \%$ des propriétés antimalariques.

\section{Cibles parasitaires de la chloroquine}

Trois voies, principalement, ont été explorées dans la recherche d'un récepteur parasitaire à la chloroquine. Elles correspondent à trois cibles potentielles: (a) l'ADN parasitaire; (b) la porphyrine du pigment malarique; (c) la vacuole digestive.

La chloroquine présente des propriétés intercalantes vis-à-vis de la double hélice de l'ADN [5]. Cette propriété est d'ailleurs partagée avec d'autres schizonticides (quinine, mépacrine), la méfloquine faisant exception. Il mécanisme d'action sélectif de ces molécules pour le parasite intra-érythrocytaire, en proposant leur intercalation dans l'ADN parasitaire. Néanmoins, la constante d'affinité faible que présente la chloroquine dans cette interaction $\left(10^{4} \mathrm{M}^{-1}\right)$, son absence d'effets rapides sur la biosynthèse des protéines, ont conduit à écarter l'intervention des propriétés intercalantes dans le mode d'action des amino-4 quinoléines.

Dès 1964, l'interaction chloroquine (CQ) ferriprotoporphyrine IX (FP) était mise en évidence, mais elle ne fut reprise et analysée qu'en 1980. L'élément le plus remarquable dans cette observation est la similitude existant entre la constante d'affinité de la chloroquine pour la porphyrine et celle de la chloroquine pour l'hématie parasitée [6]. Une hypothèse séduisante fut alors élaborée, où l'activité antimalarique de la chloroquine est liée aux effets biolo- giques du complexe CQ-FP, en particulier à ses propriétés lytiques sur les membranes. La chloroquine agirait en détournant la porphyrine de sa forme de détoxification dans le parasite (le pigment malarique) et lyserait les membranes du parasite. La spécificité d'action est bien sûr liée à l'existence de porphyrine libre dans une cellule. La résistance est, dans cette hypothèse, reliée à l'absence de pigmentation observée chez certaines de ces souches, absence due, selon les auteurs, à une chute de l'activité protéolytique chez ces mêmes souches.

Néanmoins cette approche est en contradiction avec des observations précises. En premier lieu, l'effet de la chloroquine sur le parasite ne se traduit jamais par l'observation de lyse de membranes, en particulier celles de la vacuole parasitaire contenant le pigment malarique. En deuxième lieu, il est parfaitement 
démontré que la dégradation de l'hémoglobine est toujours effective dans les souches résistantes, tant chez $P$. berghei que chez $P$. falciparum (Plasmodium humain implique dans les formes sévères de paludisme). L'étude de la structure du complexe CQ-FP par RMN [7] a montré que l'interaction chloroquine/ferriprotoporphyrine IX est une simple intercalation du noyau quinoléique entre les plans porphyriques. La nature des forces de liaison (interaction hydrophobe), la géométrie du complexe, la faible spécificité de l'interaction, ne permettent pas d'expliquer les relations structure/activité existant dans la série aminoquinoléine.

La chloroquine possède des propriétés anti-inflammatoires qui en font un médicament utilisé, par exemple, dans le traitement de l'arthrite rhumatoïde [8]. Ces propriétés seraient liées à une inhibition des fonctions lysosomiales. Il a été montré, en effet, que la chloroquine présente une remarquable affinité pour les lysosomes grâce à ses fonctions basiques [9]. Sa concentration dans les lysosomes cellulaires se traduirait par une élévation du $\mathrm{pH}$ de ces structures et donc par une perturbation de leurs fonctions enzymatiques. Le mécanisme de la concentration des bases faibles dans des compartiments cellulaires acides implique les propriétés hydrophobes des membranes qui assurent une libre diffusion des molécules neutres (non chargées), et sont imperméables aux espèces polaires (chargées) telles que les formes protonées des bases. Les bases faibles sont donc retenues dans les compartiments acides en fonction du gradient de $\mathrm{pH}$ existant entre ceuxci et les structures externes (figure 4). Dès 1972, Homewood et al. [10] proposaient que les activités antimalariques de la chloroquine soient liées à ses propriétés lysosomotropes et qu'elle agisse en inhibant la digestion de l'hémoglobine dans les vacuoles digestives supposées être les compartiments acides des parasites. Depuis, de nombreux éléments sont venus compléter et préciser cette hypothèse. L'existence de compartiments acides parasitaires est bien établie par des approches variées, qui conduisent à proposer un $\mathrm{pH}$ voisin de 5 pour ces structures. Il vient $\mathrm{m} / \mathrm{s} n^{\circ} 10$ vol. 5 , décembre 89 d'être montré, par une analyse ultrastructurale, que ces compartiments coïncident avec les vacuoles digestives du parasite [12]. Les bases faibles, telles que le chlorure d'ammonium, sont capables de déplacer la chloroquine de parasites préalablement chargés en cette molécule [13]. L'accumulation de la chloroquine dans les parasites est dépendante du $\mathrm{pH}$ extracellulaire. Enfin, une détermination précise de leur localisation intracellulaire par une détection ultrastructurale permet de montrer

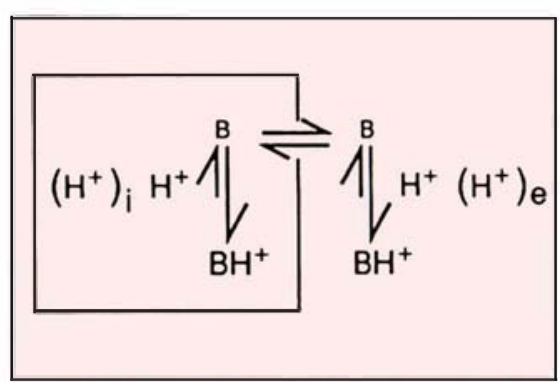

Figure 4. Mécanisme de concentration des bases faibles dans un compartiment cellulaire acide. $B$. Forme neutre de la base diffusant librement à travers la membrane. La membrane est imperméable à la forme monoprotonée $\mathrm{BH}^{+}$. Dans le cas où le pK de la base est nettement supérieur aux $\mathrm{pH}$ des deux compartiments, on montre que le rapport des concentrations en base totale $B_{T}\left(\mathrm{BH}^{+}+B\right)$ dans les compartiments externes et internes égale le rapport des concentrations en protons des compartiments :

$\left[B_{T}\right] i /\left[B_{T}\right] e=\left[\mathrm{H}^{+}\right] i /\left[\mathrm{H}^{+}\right] e$

Pour une dibase (cas de la chloroquine) on obtient :

$\left[B_{T}\right] i /\left[B_{T}\right] e=\left(\left[\mathrm{H}^{+}\right] \mathrm{i} /\left[\mathrm{H}^{+}\right] e\right)^{2}$

La membrane serait perméable à la forme monoprotonée de la chloroquine.

que la cible privilégiée des amino-4 quinoléines est la vacuole digestive du parasite lorsqu'elle dégrade l'hémoglobine [14]. La principale difficulté soulevée par cette hypothèse est de ne pouvoir prendre en compte la spécificité antiparasitaire des aminoquinoléines. En effet ces drogues sont capables de perturber le métabolisme du parasite à des doses 100 à 1000 fois plus faibles que celles efficaces sur des cellules de mammifères [15].

Aucune des hypothèses proposées ici n'est ainsi réellement satisfaisante dans l'explication des mécanismes d'action des antimalariques. L'étude des mécanismes de résistances a cependant permis, ces dernières années, de mettre en évidence quelques aspects nouveaux des relations parasite/chloroquine.

\section{La résistance}

La production de souches résistantes à partir des souches virulentes a été obtenue par une simple pression médicamenteuse sur un modèle de plasmodium de rongeur comme $P$. berghei [16]. Des études comparatives furent entreprises, dès les premiers travaux, sur le mécanisme d'action entre souches sensibles et souches résistantes. Il fut ainsi montré que les souches résistantes accumulent moins de chloroquine que les souches sensibles [17] ; cela se traduit par une chute de la constante d'affinité de la drogue pour le parasite. Dans le cas de P. falciparum, les études réalisées in vitro démontrent un phénomène identique [26]. Des éléments récents sont venus préciser les mécanismes mis en jeu par le parasite pour résister aux aminoquinoléines. Une étude précise de localisation intracellulaire des quinoléines sur des souches de $P$. berghei sensibles et résistantes montre que la résistance est associée à une dispersion des molécules dans le cytoplasme parasitaire [12]. Bien que comportant toujours des compartiments acides, le parasite résistant est incapable de retenir les bases faibles (quinoléiques ou autres) dans ces structures [12]. Il est possible de proposer, dans ce cas, un mode de résistance aux amino-4 quinoléines qui ferait intervenir une déficience des mécanismes de ré-acidification des vacuoles parasitaires dans lesquelles se sont accumulées des bases faibles. Un mécanisme totalement différent vient d'être proposé dans le cas de $P$. falciparum. Il se rapproche des mécanismes rencontrés dans la polychimiorésistance de cellules leucémiques humaines aux anticancéreux tels que vinblastine, vincristine, daunorubicine. La chloroquine se révèle capable de restituer partiellement la sensibilité à ces cytotoxiques des cellules cancéreuses résistantes [19]. Elle partage cette propriété avec le véra- 


\section{RÉFÉRENCES}

15. Krogstad DJ, Schlesinger PH. A perspective on antimalarial action : effects of weak bases on Plasmodium falciparum. Biochem Pharmacol 1986 ; 35 : 547-2.

16. Macomber PB, O'Brien RL, Hahn FE. Chloroquine: physiological basis of drug resistance in $P$. berghei. Science $1966 ; 152$ : 1374-5.

17. Warhurse DC, Thomas SC. Pharmacology of the malaria parasite : a study of doseresponse relationships in chloroquine-induced autophagic vacuole formation in Plasmodium berghei. Biochem Pharmacol 1975 ; 24 : 2047-56.

18. Geary TG, Jensen JB, Ginsburg H. Uptake of $3 \mathrm{H}$ chloroquine by drug-sensitive and -resistant strains of the human malaria parasite Plasmodium falciparum. Biochem Pharmacol 1986 ; 35 : 3805-12.

19. Zamora JM, Beck WT. Chloroquine enhancement of anticancer drug cytotoxicity in multiple drug resistant human leukemic cells. Biochem Pharmacol 1986 ; 35 : 4303-10.

20. Zamora JM, Pearce HL, Beck WT. Physical-chemical properties shared by compounds that modulate multidrug resistance in human leukemiccells. Mol Pharmacol 1988 ; 33 : 45462.

21. Martin SK, Odula AMJ, Milhous WK. Reversal of chloroquine resistance in Plasmodium falciparum by verapamil. Science 1987 ; 235 : 899-901.

22. Krogstad DJ, Gluzman IY, Kyle DE, et al. Efflux of chloroquine from Plasmodium falciparum: mechanism of chloroquine resistance. Science $1987 ; 238$ : 1283-5.

23. YeZG, Van Dyke K. Reversal of chloroquine resistance in falciparum malaria independent of calcium channels. Biochem Biophys Res Commun 1988; 155 : 476-81.

24. Fitch CD, Warhurst DC. The mode of action of chloroquine and related malarial schizontocides. Parasitol Today 1986; 12 : 329-34.

25. Mikkelsen RB, Tanabe K, Wallach FH. Membrane potential of plasmodium-infected erythrocytes. J Cell Biol 1982 ; 93 : 685-9.

26. Mikkelsen RB, Wallach DFH, Van Doren E, Nillni EA. Membrane potential of erythrocytic stages of Plasmodium chabaudi free of the host cell membrane. Mol Biochem Parasitol 1986 ; 21 : 83-92.

27. Bitonti AJ, Sjoerdsma A, McCann PP, et al. Reversal of chloroquine resistance in malaria parasite Plasmodium falciparum by pamil (un inhibiteur des canaux calciques, présentant des propriétés lysosomotropes) et quelques alcaloïdes [20]. De même, le vérapamil s'est avéré capable de restituer la sensibilité à la chloroquine de souches plasmodiales chloroquino-résistantes [21]. Cet effet se traduit par une inhibition d'un mécanisme d'évasion de ces molécules de leurs cellules cibles [22], il n'est pas lié chez Plasmodium à l'inhibition des canaux calciques [23]. Il pourrait faire intervenir, comme dans le cas des cellules cancéreuses, une glycoprotéine membranaire, contrôlant les concentrations intracellulaires de nombreuses drogues par un mécanisme de transport extracellulaire.

Les deux mécanismes mentionnés font intervenir une fuite de la chloroquine, soit au niveau de son site d'action dans le parasite, soit au niveau global du parasite. Ils diffèrent au niveau des mécanismes cellulaires hypothétiques impliqués dans cette évasion. $\mathrm{Ni}$ l'existence d'une glycoprotéine membranaire capable d'exporter la chloroquine, ni l'existence d'ATPases membranaires n'ont pu être directement mises en évidence à ce jour chez Plasmodium. La localisation de cette hypothétique glycoprotéine dans la membrane vacuolaire du parasite et dans la membrane cytoplasmique pourrait pourtant expliquer l'ensemble des données expérimentales issues de ces divers travaux. Sa mise en évidence constitue un objectif important pour

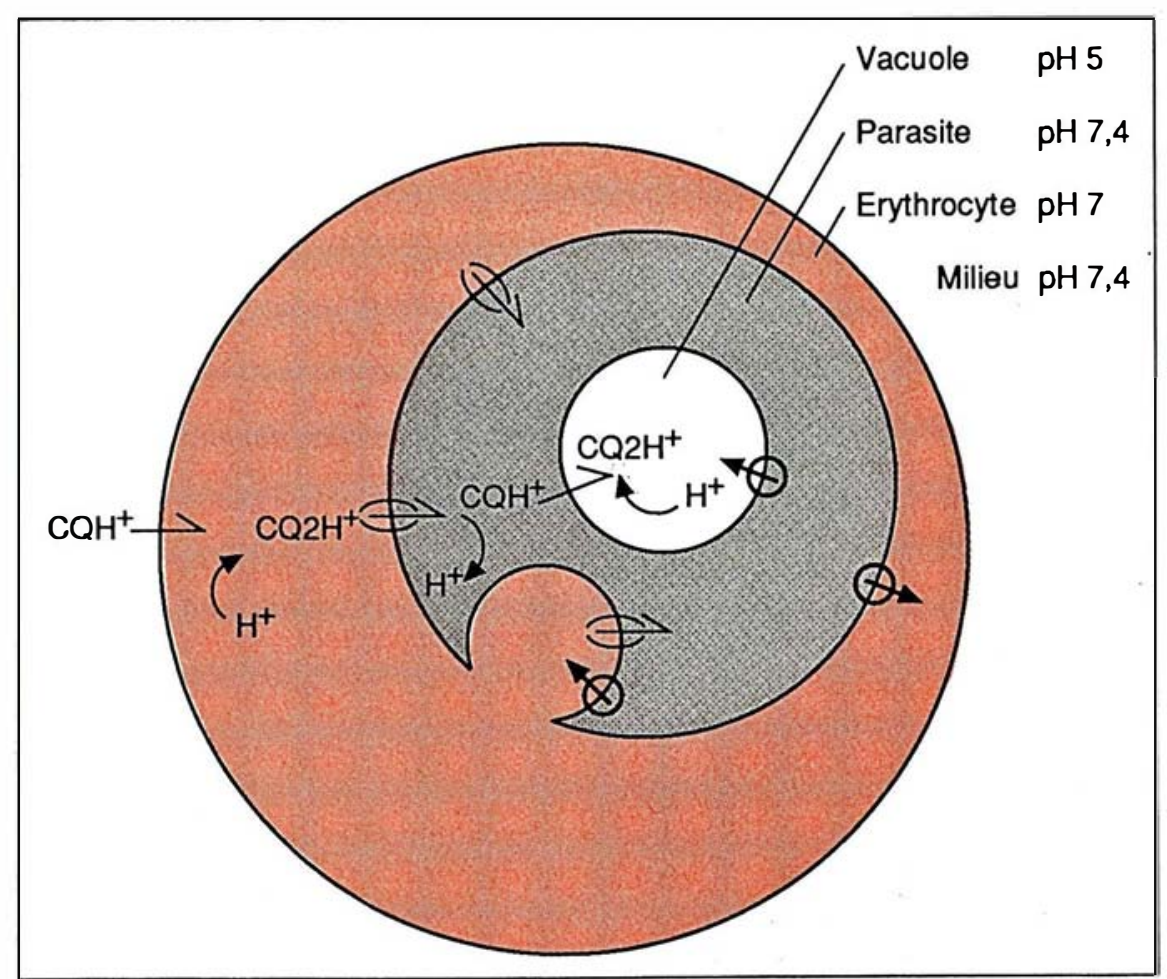

Figure 5. Profil de pH des divers compartiments d'un érythrocyte parasité et hypothèse d'un transport actif de la chloroquine par le parasite. La chloroquine (CQ) est majoritairement sous forme protonée au $\mathrm{pH}$ physiologique (0,2 \% seulement sous forme neutre). La membrane est supposée perméable à la forme monoprotonée $\mathrm{CQH}^{+}$mais imperméable à la forme diprotonée $\mathrm{CQ}_{2} \mathrm{H}^{+}$. Le passage de la forme $\mathrm{CQ}_{2} \mathrm{H}^{+}$(contre le gradient de $\mathrm{pH}$ ) est assuré par la perméase ( $)$ ) fonctionnant grâce à un gradient de proton maintenu par des $\mathrm{H}^{+}$ATPases ( $७$ ). L'existence de cette même perméase dans la membrane de la vacuole permet d'expliquer la chute de concentration de la chloroquine dans ce compartiment et son accumulation dans le cytoplasme parasitaire. 
la compréhension du (des) mode(s) de résistance mis en place par le parasite.

\section{Propriétés non basiques de la chloroquine}

Le mécanisme d'action antimalarique de la chloroquine est loin d'être complètement élucidé. L'explication d'un phénomène clé tel que la sélectivité pour les formes érythrocytaires du parasite n'est pas prise en compte par les trois hypothèses développées précédemment, pas plus que ne sont pris en compte les derniers éléments sur les mécanismes de résistance développés par le parasite. Une hypothèse récemment introduite par D. Warhurst [24] essaie de rassembler tous ces éléments. Cet auteur propose l'intervention d'une perméase, d'un transport actif de la chloroquine dans le parasite, dont la distribution dans la membrane cytoplasmique et vacuolaire contrôle la distribution des drogues dans le parasite et donc la sensibilité à la chloroquine (figure 5). Il faut noter que les travaux sur les $\mathrm{pH}$ intracellulaires des érythrocytes parasités impliquent qu'à une des étapes de pénétration, la chloroquine franchisse la barrière cytoplasmique contre un gradient de proton [25]. En effet, une analyse des potentiels membranaires [26] des formes érythrocytaires conduit à l'établissement d'un profil de $\mathrm{pH}$, entre milieu externe et vacuole parasitaire, où le $\mathrm{pH}$ du cytoplasme parasitaire est maintenu alcalin par rapport à celui du globule rouge (figure 5). Il est, dans ces conditions, difficile de concilier ces données avec un mécanisme de concentration impliquant les propriétés lysosomotropes de la chloroquine.

Cette analyse suggère que les propriétés basiques des quinoléines ne sont pas seules responsables de l'activité antimalarique des schizonticides sanguins, et qu'interviennent d'autres facteurs liés à la structure des molécules et (ou) à des facteurs parasitaires, en particulier au niveau des mécanismes de concentration intraparasitaire des schizonticides. En revanche, les propriétés lysosomotropes seraient impliquées dans les activités anti-inflammatoires et les effets secondaires de la chloroquine. Elle est en effet utilisée dans ces circonstances à des doses nettement plus importantes que les doses antimalariques [9].

\section{Conclusion}

Si les mécanismes responsables de la concentration intraparasitaire de la chloroquine sont encore mal connus, la cible primitive des aminoquinoléines chez Plasmodium (la vacuole digestive) est parfaitement identifiée. L'activité de la chloroquine sur cette structure se traduit par la formation de la vacuole autophage, mais les mécanismes intervenant dans cet événement ne sont pas connus. L'alcalinisation de la vacuole par la chloroquine fait toujours l'objet d'investigations, et les métabolismes vacuolaires potentiellement perturbés par cette drogue sont variés: protéases intervenant dans la dégradation de l'hémoglobine, phospholipases, transit des protéines parasitaires?

La remarquable efficacité antimalarique de la chloroquine reste largement inexpliquée, mais son usage est menacé par le développement de la résistance. Une expérimentation récente vient de montrer les potentialités des voies de recherches entreprises. La désipramine, un antidépresseur, inhibiteur des canaux calciques, s'est révélé capable de restituer la sensibilité à la chloroquine de souches chloroquino-résistantes. Cette étude a été réalisée in vivo chez le singe Aotus à des concentrations plasmatiques en drogues compatibles avec un usage clinique[27]

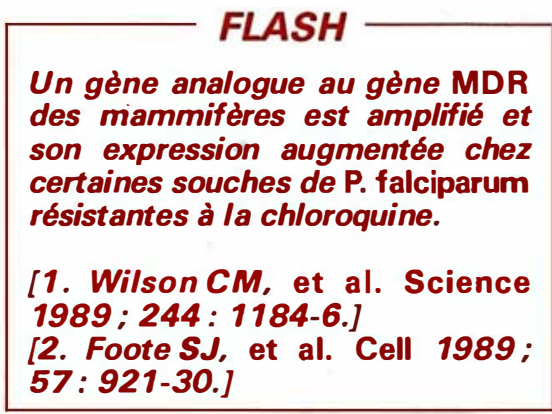

\section{Summary}

Chloroquine : antimalarial mode of action and resistance

Chemotherapy and chemoprophylaxy are essential for the treatment of acute malaria infections. The spread of resistance to chloroquine of Plasmodium falciparum, the major drug used for the treatment, has been a serious problem since 1960. Design of replacement drugs may be obtained through knowledge of their mode of action and mechanisms of resistance. The selective toxicity of antimalarial blood schizonticides is based on concentrative uptake by infected erythrocytes. A major hypothesis has been advanced to account for the biological properties of chloroquine; this drug act as a lysosomotropic agent, accumulating into acidic intracellular compartments by virtue of its weak base properties. However malaria parasites are affected at concentrations 100 1000 times less concentrated than those effective in mammalian cells. This suggests that the parasite has a drug uptake mechanism which concentrate chloroquine beyond that due to its properties as a weak base. It has been shown that drug-resistant strains of malaria take up chloroquine less efficiently. Mechanisms are related either to a release of the drug in the cytoplasm of the parasite or to the efflux of chloroquine from the resistant cell mediated through a carrier as observed for the multidrug resistance in cancer chemotherapy. Verapamil, a calcium channel blocker, reverse chloroquine resistance by inhibiting its efflux from the resistant parasite.

\section{TIRÉS A PART}

\section{S. Moreau.}

